\title{
Antisense Oligonucleotides to Cux-1, a Cut-related Homeobox Gene, Cause Increased Apoptosis in Mouse Embryonic Kidney Cultures
}

\author{
Susan E. Quaggin, ${ }^{\star}$ Herman Yeger, ${ }^{\ddagger}$ and Peter Igarashi* \\ *Department of Internal Medicine, Yale University School of Medicine, New Haven, Connecticut 06520; and ${ }^{\ddagger}$ Department of Pathology, \\ The Hospital for Sick Children, University of Toronto, Toronto, Canada M5G 1 X8
}

\begin{abstract}
Cux-1 is a murine homeobox gene that is highly and transiently expressed in the developing kidney. To further evaluate the role of Cux-1 in mammalian kidney development, organotypic cultures of embryonic mouse kidney were incubated with phosphorothioate-coupled antisense Cux-1 oligonucleotides (ODNs) in the presence of cationic liposomes. Inhibition of $\mathrm{Cux}-1$ expression by antisense ODNs was verified by reverse transcription-PCR. Metanephroi that were incubated with antisense Cux-1 ODNs were $23 \%$ smaller than metanephroi that were incubated with sense Cux-1 ODNs. Morphologic analysis of metanephroi that were treated with antisense $C u x-1$ ODNs revealed that ureteric buds and induced epithelial structures were present. However, extensive areas of cell death containing shrunken cells with pyknotic nuclei were also evident. The presence of increased apoptosis was verified by ultrastructural and terminal transferase-mediated dUTP nick end labeling analyses. Two different antisense Cux-1 ODNs targeting either the translation start codon or the homeobox produced increased apoptosis. In contrast, metanephroi incubated with sense ODNs exhibited only occasional apoptotic cells. We conclude that the presence of antisense Cux-1 ODNs does not block nephron induction, but results instead in increased apoptosis. Proper regulation of Cux-1 expression may be necessary for normal kidney development. (J. Clin. Invest. 1997. 99:718-724.) Key words: organ culture - fetal development $\cdot$ homeodomain proteins - programmed cell death • transcription factors
\end{abstract}

\section{Introduction}

The definitive kidney in mammals, the metanephros, begins to develop during the fifth week of gestation in humans and $11.5 \mathrm{~d}$ postcoitus (d.p.c.) ${ }^{1}$ in the mouse (1). The metanephros has a

Portions of this work have appeared in abstract form (1995. Mol. Biol. Cell. 6:326a).

Address correspondence to Peter Igarashi, Section of Nephrology, Yale University School of Medicine, 333 Cedar Street, New Haven, CT 06520-8029. Phone: 203-785-7111; FAX: 203-785-7068; E-mail: peter.igarashi@yale.edu

Received for publication 10 September 1996 and accepted in revised form 27 November 1996.

1. Abbreviations used in this paper: d.p.c., days postcoitus; ODN, oligodeoxyribonucleotide; RT-PCR, reverse transcription-PCR; TUNEL, terminal transferase-mediated dUTP nick end labeling.

J. Clin. Invest.

(C) The American Society for Clinical Investigation, Inc. 0021-9738/97/02/0718/07 \$2.00

Volume 99, Number 4, February 1997, 718-724 dual origin from the ureteric bud and the metanephric blastema. During the development of the metanephros, the ureteric bud emerges from the caudal end of the mesonephric duct and invades the metanephric blastema. In response to an inductive signal from the branching ureteric bud, mesenchymal cells comprising the metanephric blastema condense and undergo epithelial differentiation forming a renal vesicle. The renal vesicle invaginates to form an $S$-shaped body that is the primordium of the glomerulus, proximal tubule, loop of Henle, and distal tubule. The collecting ducts, renal pelvis, and ureter are derived from the ureteric bud. Nephrogenesis involves multiple developmental processes including stem-cell differentiation, induction, mesenchymal-to-epithelial transformation, pattern formation, and apoptosis (2,3). During nephrogenesis, the expression of many genes is temporally and spatially regulated. However, only a few transcription factors that are essential for kidney development have been identified (4-6).

Homeobox genes encode transcription factors that regulate developmental gene expression in many organisms. Cux-1 is a murine homeobox gene that is related to the Drosophila cut gene (7). During mouse embryogenesis, Cux-1 is expressed in the developing excretory system including the mesonephros, mesonephric duct, and metanephros (8). In the developing metanephros, $C u x-1$ is highly expressed at 13.5 d.p.c., which is shortly after metanephric induction commences, and expression remains high until the completion of nephrogenesis $\sim 1$ wk postpartum. Thereafter, expression of Cux-1 decreases such that only low levels of expression are detected in the adult kidney. Studies using in situ hybridization have shown that Cux-1 is highly expressed in metanephric mesenchyme, induced epithelial structures (renal vesicle, $S$-shaped bodies), and ampullae of ureteric buds (8). Expression of Cux-1 is downregulated during terminal differentiation, and $C u x-1$ is not detectable by in situ hybridization in mature tubules or glomeruli. This temporally and spatially restricted pattern of expression suggests that $C u x-1$ may play a role in kidney development.

Organotypic cultures of metanephric explants represent an excellent in vitro model for monitoring developmental processes during early nephrogenesis. When metanephroi are removed from embryos and placed in appropriate media, they continue to develop in vitro and display many of the processes of nephron induction and differentiation that are observed in vivo (9-11). By incubating metanephric explants with antisense oligodeoxyribonucleotides (ODNs), the expression of specific genes can be inhibited and the consequences to kidney development can be monitored. Recently, the functions of Pax-2 and nerve growth factor receptor were studied in the developing kidney using antisense ODNs. Pax-2 is a paired-box protein that is highly expressed in condensed mesenchyme, $S$-shaped bodies, and ureteric ducts. Incubation of metanephric kidney cultures with antisense Pax-2 ODNs resulted in a block in nephrogenesis, and subsequent studies using gene 
targeting verified that Pax-2 null mice failed to form kidneys or ureters $(5,12)$. Antisense inhibition of nerve growth factor receptor in organotypic kidney cultures perturbed both epithelial differentiation and branching morphogenesis (13). In this paper, we report the use of antisense ODNs in organotypic metanephric cultures to examine the role of $C u x-1$ in kidney development.

\section{Methods}

Antisense oligonucleotides. 19-mer antisense ODNs were designed to target the translation start codon of $\mathrm{Cux}-1$ (ASTr) corresponding to nucleotides 321-340 (accession number X75013; Genbank/EMBL/ DDBJ) or the homeobox (ASH) corresponding to nucleotides 35283547. The sequence of ASTr was 5'-TTTGAGGTGGTGGACATCT-3', and the sequence of ASH was 5'-TATGCTCGCTTCAGCGCTT-3'. As negative controls, sense ODNs (STr, SH) were synthesized to the identical regions. The sequence of STr was 5'-AGATGTCCACCACCTCAAA-3', and the sequence of SH was 5'-AAGCGCTGAAGCGAGCATA-3'. Oligonucleotides were phosphorothioatecoupled and synthesized by the Yale Program in Critical Technologies. The ODNs were purified by HPLC to $>99 \%$ purity.

Organotypic kidney cultures. Organotypic kidney cultures were established from mouse embryos essentially as described previously (14). Embryos were dissected from timed-pregnant mice at 12.5 d.p.c. Embryonic age was verified according to Theiler's criteria (15). Metanephroi and associated ureteric buds were microdissected en bloc and placed in holding medium (L15 medium supplemented with $1 \times$ $\mathrm{MITO}+$ serum extender). All media were supplemented with $100 \mathrm{U}$ penicillin $/ \mathrm{ml}$ and $0.1 \mathrm{mg}$ streptomycin $/ \mathrm{ml}$.

Media containing antisense or sense ODNs were prepared as follows: $80 \mu \mathrm{l}$ of cationic liposomes (LipofectAmine; Life Technologies, Inc., Gaithersburg, MD) was added to $100 \mu$ of prewarmed $\left(37^{\circ} \mathrm{C}\right)$ Opti-MEM(Life Technologies, Inc.,). In a separate tube, an antisense ODN (ASTr or ASH) or a sense ODN (STr or SH) was added to 100 $\mu 1$ of Opti-MEM to give a final concentration of $5 \mu \mathrm{M}$ ODN in $2 \mathrm{ml}$. The LipofectAmine and ODN mixtures were combined and preincubated for $45 \mathrm{~min}$ at room temperature. The LipofectAmine/ODN mixture was then added to DMEM-F12 medium supplemented with $5 \times \mathrm{MITO}+$ to give a final volume of $2 \mathrm{ml}$ that was then added to each well of a six-well tissue culture plate containing Cyclopore (polyethylene terphthalate; Falcon Labware, Cockeysville, MD) inserts. Metanephroi were transferred from the holding media onto the surface of the Cyclopore membranes. For each treatment with antisense and sense ODNs, metanephroi from the same embryo were paired to control for any variability in gestational age. Cultures were incubated at $37^{\circ} \mathrm{C}$ under an atmosphere of $5 \% \mathrm{CO}_{2}$. After $24 \mathrm{~h}$, the growth medium was replaced with fresh LipofectAmine and ODN mixtures prepared as described previously. After $52 \mathrm{~h}$, the growth medium was supplemented with $10 \%$ fetal bovine serum. Metanephroi were examined daily by light microscopy and photomicrographs were obtained using a microscope (CK-2; Olympus Corp., Lake Success, NY). After $68 \mathrm{~h}$, the metanephroi were washed briefly in PBS and fixed for morphology or terminal transferase-mediated dUTP nick end labeling (TUNEL) assay.

An additional 20 metanephroi were treated with antisense or sense ODNs in the absence of LipofectAmine. These metanephric explants were treated in an identical fashion to those described above, except that $10 \mu \mathrm{M}$ antisense (ASTr) or sense (STr) ODN was added to a final volume of $2 \mathrm{ml}$ in DMEM-F12 supplemented with $5 \times$ MITO + and antibiotics. Media were changed daily with addition of fresh ODN each time. Metanephroi were cultured for a total of $96 \mathrm{~h}$ and fixed.

Reverse Transcription-PCR. Reverse transcription of RNA and PCR amplification of cDNA (RT-PCR) was performed using the
rTth Reverse Transcriptase RNA PCR Kit from Perkin Elmer Cetus Instruments (Foster City, CA). 11 sense and 11 antisense ODNtreated metanephroi were grown in culture for $68 \mathrm{~h}$. Total RNA was purified using acid guanidinium thiocyanate-phenol-chloroform extraction (16). First strand cDNA synthesis was performed in a total volume of $20 \mu \mathrm{l}$ containing $10 \mathrm{mM}$ Tris- $\mathrm{Cl}, \mathrm{pH} 8.3,100 \mathrm{mM} \mathrm{KCl}, 1 \mathrm{mM}$ $\mathrm{MnCl}_{2}, 200 \mu \mathrm{M}$ of each dNTP, $0.5 \mathrm{U}$ of rTth DNA polymerase, $750 \mathrm{nM}$ of an antisense Cux-1-specific primer (5'-TGGTTGGCTCGTTCAAGGTCGGTCA-3'), and $165 \mathrm{ng}$ of RNA. Reverse transcription was carried out by incubation at $65^{\circ} \mathrm{C}$ for 9 min, followed by incubation at $70^{\circ} \mathrm{C}$ for $6 \mathrm{~min}$. The reaction was stopped by placing the tubes on ice, and $80 \mu \mathrm{l}$ of a PCR amplification reaction mix was added to give final concentrations of $8 \mathrm{mM}$ Tris-Cl, $\mathrm{pH} 8.3,4 \%$ glycerol, $80 \mathrm{mM} \mathrm{KCl}, 0.04 \%$ Tween 20, $0.6 \mathrm{mM}$ EGTA, $1.5 \mathrm{mM} \mathrm{MgCl}_{2}$, and $150 \mathrm{nM} \mathrm{Cux-1-specific}$ sense primer (5'-TCAGGCCGAGACCATTGCTCTGGA-3'). PCR amplification was performed in a thermal cycler (Perkin Elmer Cetus Instruments) by incubation at $95^{\circ} \mathrm{C}$ for $120 \mathrm{~s}$ followed by 30 cycles of incubation at $95^{\circ} \mathrm{C}$ for $60 \mathrm{~s}$ and $60^{\circ} \mathrm{C}$ for $60 \mathrm{~s}$. After a final incubation at $60^{\circ} \mathrm{C}$ for $7 \mathrm{~min}$, samples were electrophoresed on a $3 \%$ NuSieve agarose gel, and products were visualized by ethidium bromide staining.

Morphologic studies. Metanephroi were fixed for $20 \mathrm{~min}$ at room temperature in PBS containing $1.5 \%$ glutaraldehyde and 5\% sucrose. Metanephroi were postfixed in $\mathrm{OsO}_{4}$ reduced with potassium ferrocyanide and embedded in epon. 1- $\mu \mathrm{m}$-thick sections were cut on a Leica Ultracut E ultramicrotome, stained with methylene blue and azure II, and then examined by light microscopy. Samples for electron microscopy were fixed similarly, embedded in epon, and cut into ultrathin (80-nm) sections. Sections were stained with lead citrate and examined with an Oberkochen electron microscope (D-7082; Carl Zeiss, Inc., Thornwood, NY).

For quantification of metanephric size, metanephroi treated with sense and antisense ODNs were photographed under light microscopy at identical magnification. Images were digitized using a film scanner (LS-1000; Nikon Inc., Melville, NY). Metanephroi were outlined manually, and the surface areas were calculated by pixel counting. The mean sizes of sense and antisense ODN-treated metanephroi were calculated for each experiment and compared using Student's two-tailed paired $t$ test. Statistical significance was defined as $P<0.05$.

TUNEL assay. Terminal transferase-mediated dUTP nick end labeling (17) was performed using the Fluorescein In Situ Cell Death Detection Kit from Boehringer Mannheim Biochemicals (Indianapolis, IN) according to the manufacturer's directions and as previously described (18). Briefly, metanephroi were fixed in $4 \%$ paraformaldehyde at room temperature for $20 \mathrm{~min}$ and cryoprotected overnight with $30 \%$ sucrose in PBS. 8- $\mu \mathrm{m}$-thick cryosections were prepared using a Jung Frigocut cryostat (2800N; Leica Inc., Deerfield, IL). Sections were washed three times in PBS, permeabilized for $2 \mathrm{~min}$ on ice in PBS containing $0.1 \%$ sodium citrate and $0.1 \%$ Triton X-100, and then rinsed twice in PBS. Sections were overlaid with $50 \mu$ l of labeling solution containing $30 \mathrm{mM}$ Tris- $\mathrm{Cl}, \mathrm{pH} 7.2,140 \mathrm{mM}$ sodium cacodylate, $1 \mathrm{mM} \mathrm{CoCl}_{2}, 0.3 \mathrm{nmol}$ fluorescein-12-dUTP, $3 \mathrm{nmol} \mathrm{dATP}$, and $25 \mathrm{U}$ terminal transferase. Slides were covered with a glass coverslip, incubated for $1 \mathrm{~h}$ at $37^{\circ} \mathrm{C}$ in the dark, and then rinsed three times with PBS. Sections were mounted with Vectashield (Vector Laboratories, Inc., Burlingame, CA), and fluorescence microscopy was performed using a fluorescent microscope (Carl Zeiss, Inc.).

Materials. Timed-pregnant mice (strain CD-1) were from Charles River Laboratories (Wilmington, MA). The morning of detection of the copulatory plug was designated 0.5 d.p.c. Tissue culture growth media were from Life Technologies, Inc. MITO+ was from Collaborative Biomedical Inc. (Chicago, IL) and other media supplements were from Sigma Chemical Co. (St. Louis, MO). Agarose was from FMC BioProducts (Rockland, ME). Glutaraldehyde was from Electron Microscopy Sciences (Ft. Washington, PA). Other chemicals were of analytical or molecular biology grade from Baker (Sanford, ME) or Sigma Chemical Co. 


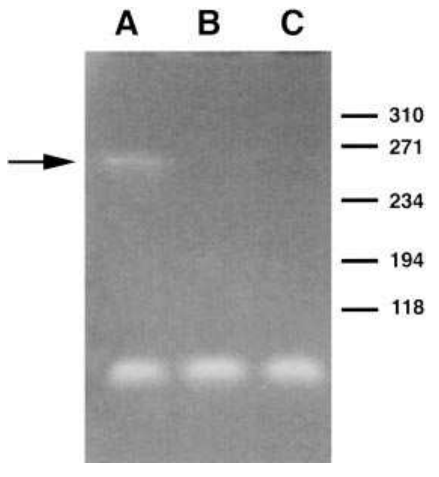

Figure 1. Expression of Cux-1 in metanephroi. RT-PCR was performed using $165 \mathrm{ng}$ of total RNA isolated from metanephroi after incubation with sense Cux-1 ODN $(A)$ or antisense Cux-1 ODN ( $B$ and $C)$. PCR products were separated in a $3 \%$ agarose gel, stained with ethidium bromide, and a 258-bp product of the predicted size (arrow) was observed in the sense ODN-treated metanephroi only. The results shown are representative of two independent experiments.

\section{Results}

RT-PCR was performed to determine whether incubation of cultured metanephroi with $C u x-1$ antisense ODNs resulted in inhibition of $C u x-1$ expression. Fig. 1 shows that a PCR product of the predicted size (258 bp) could be amplified from RNA derived from metanephroi incubated with a sense Cux-1 ODN. In contrast, no product was obtained after amplification of RNA derived from metanephroi incubated with antisense Cux-1 ODNs. These results indicated that incubation with antisense (but not sense) ODNs inhibited $C u x-1$ expression.

Antisense and sense ODN-treated metanephroi were examined daily by light microscopy. Initially, all metanephroi were matched for gestational age and had a similar appearance (Fig. 2, $A$ and $B$ ). By $48 \mathrm{~h}$, the antisense ODN-treated metanephroi were visibly smaller and showed more prominent ureteric branches (Fig. 2 F, arrowheads), which was apparently due to loss of intervening tissue. Branching of the ureteric buds and development of nephron structures were observed in both antisense and sense ODN-treated metanephroi (Fig. 2, $G$ and $H$, arrows). However, fewer nephron structures developed in the antisense ODN-treated metanephroi. Similar results were seen in metanephroi treated with ODNs in the presence of cationic liposomes (Fig. 2, $I$ and $J$ ). The presence of cationic liposomes did not visibly affect the growth or morphology of sense ODN-treated metanephroi.

The surface areas of 24 sense and 24 matched antisense ODN-treated metanephroi from five separate experiments were compared. The mean size of sense and antisense ODNtreated metanephroi from each experiment (embryos from a single mother) were paired to control for minor variations in gestational age. Fig. 3 shows that the mean size of metanephroi incubated with antisense ODNs was $1.99 \mathrm{~mm}^{2}$, which was $23 \%$ less than the mean size of metanephroi incubated with sense ODNs $\left(2.58 \mathrm{~mm}^{2}\right)$. This difference was statistically significant using Student's paired $t$ test $(P=0.025)$.

Fig. $4 A$ shows that metanephroi incubated with a sense ODN (STr) exhibited a normal pattern of differentiation in which all stages of metanephric development were visible including $S$-shaped bodies and ureteric buds (labeled $S$ and $U B$, respectively, in Fig. $4 A$ ). Fig. $4 B$ shows that metanephroi incubated with an antisense ODN (ASTr) also exhibited maturing nephron structures. However, antisense ODN-treated metanephroi also contained large regions of undifferentiatedappearing cells, many with pyknotic nuclei. These regions had
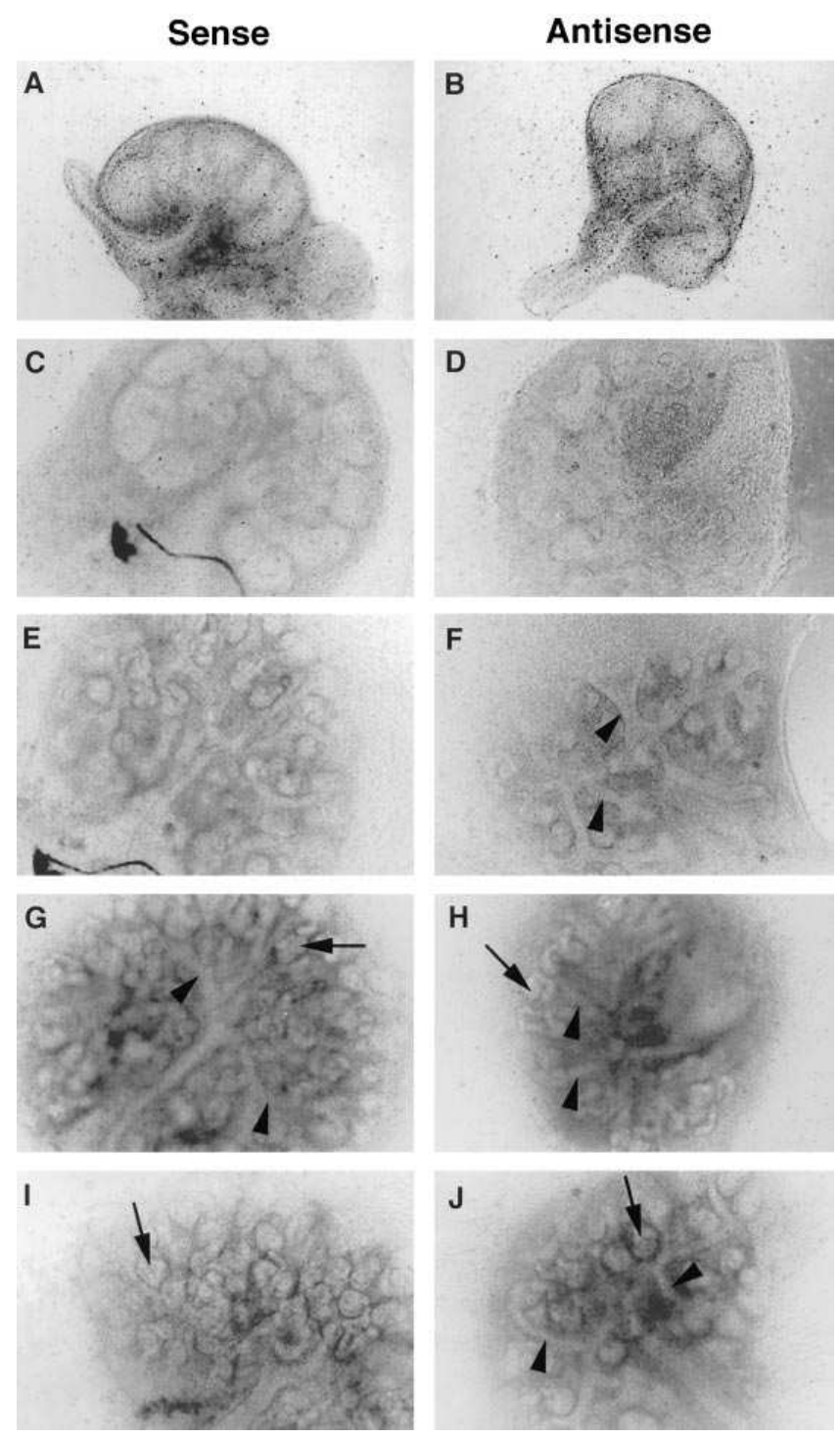

Figure 2. Morphology of metanephroi cultured in the presence of sense or antisense ODNs. Serial light photomicrographs of paired metanephric explants are shown. (Sense) Metanephros isolated from a 12.5-d.p.c. mouse embryo and incubated with $10 \mu \mathrm{M}$ sense $C u x-1$ ODN matching the translation start codon (STr) for $0(A), 24(C), 48$ $(E)$, and $96 \mathrm{~h}(G)$. (Antisense) Metanephros isolated from a 12.5d.p.c. mouse embryo and incubated with $10 \mu \mathrm{M}$ antisense $C u x-1$ ODN (ASTr) for $0(B), 24(D), 48(F)$, and $96 \mathrm{~h}(H)$. Note the crowding of ureteric branches (arrowheads) and smaller size of the antisense ODN-treated metanephros. ( $I$ and $J$ ) Photomicrographs of paired 12.5-d.p.c metanephric explants after incubation for $68 \mathrm{~h}$ with $5 \mu \mathrm{M}$ sense $C u x-1$ ODN (STr, $I$ ) or antisense ODN (ASTr, $J$ ). Delivery of ODNs to the metanephroi was facilitated by premixing with cationic liposomes. Note the prominence of ureteric branches in the antisense ODN-treated metanephros (arrowheads) due to apparent loss of intervening mesenchymal tissue and fewer overlying epithelial structures. Maturing nephric structures are indicated by arrows. $27 \times$.

fewer nephron structures, and some regions were devoid of recognizable nephric structures (Fig. 4 B, unlabeled arrow). At higher magnification, these regions contained shrunken cells with pyknotic nuclei, possibly representing apoptotic bodies (Fig. 4, $C$ and $D$ ). Incubation with $\mathrm{ASH}$, a different antisense 


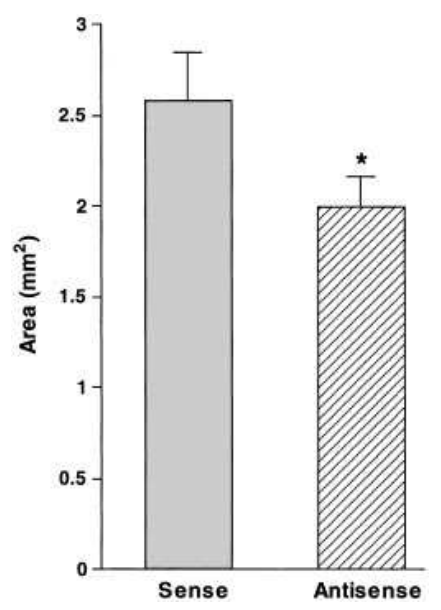

Figure 3. Comparison of sizes of metanephroi treated with sense or antisense ODNs. The mean size of 24 paired metanephroi incubated with sense (shaded bar) or antisense (hatched bar) Cux-1 ODNs are shown. Error bars represent standard errors. *Statistically different from sense control $(P=0.025)$. Results from five independent experiments are shown.

ODN targeted to the homeobox, produced similar increases in apoptotic-appearing cells compared with a homeobox sense control ODN (data not shown). The antisense ODN-treated explants were visibly smaller than sense ODN-treated explants, which may be the result of cell loss due to apoptosis.

The most characteristic biochemical feature of apoptosis is chromatin cleavage (19). The resultant nuclear DNA contains nicks that can be labeled enzymatically with fluorescein12-dUTP using terminal transferase (TUNEL) $(17,18)$. This procedure was performed to verify whether increased apoptosis was present in metanephroi after incubation with antisense ODNs. To validate the assay, we first performed TUNEL on sections of immature mouse kidney at $3 \mathrm{~d}$ postpartum. Previous studies by Coles et al. (20) indicated that apoptosis is present during normal kidney development. These authors used morphologic criteria to identify the presence of isolated apoptotic cells in the nephrogenic zone of the developing rat metanephros. Since nephrogenesis is ongoing at birth in rodents, we examined 3-d postpartum mouse kidney that contains a well-defined nephrogenic zone. Fig. $5 \mathrm{E}$ shows that isolated cells in the subcortical nephrogenic zone were labeled by the TUNEL procedure (arrows), consistent with the results of Coles et al. and verifying the validity of the assay.

Similar to the normal kidney of the developing mouse, metanephroi incubated in vitro with sense ODNs contained isolated cells that were labeled by the TUNEL method (Fig. $5 C$ and $D)$. However, metanephroi incubated with antisense ODNs exhibited a marked increase in labeled cells consistent with an increase in apoptosis (Fig. 5, $G$ and $H$ ). Due to the very high intensity of the fluorescence signal in the antisense ODN-
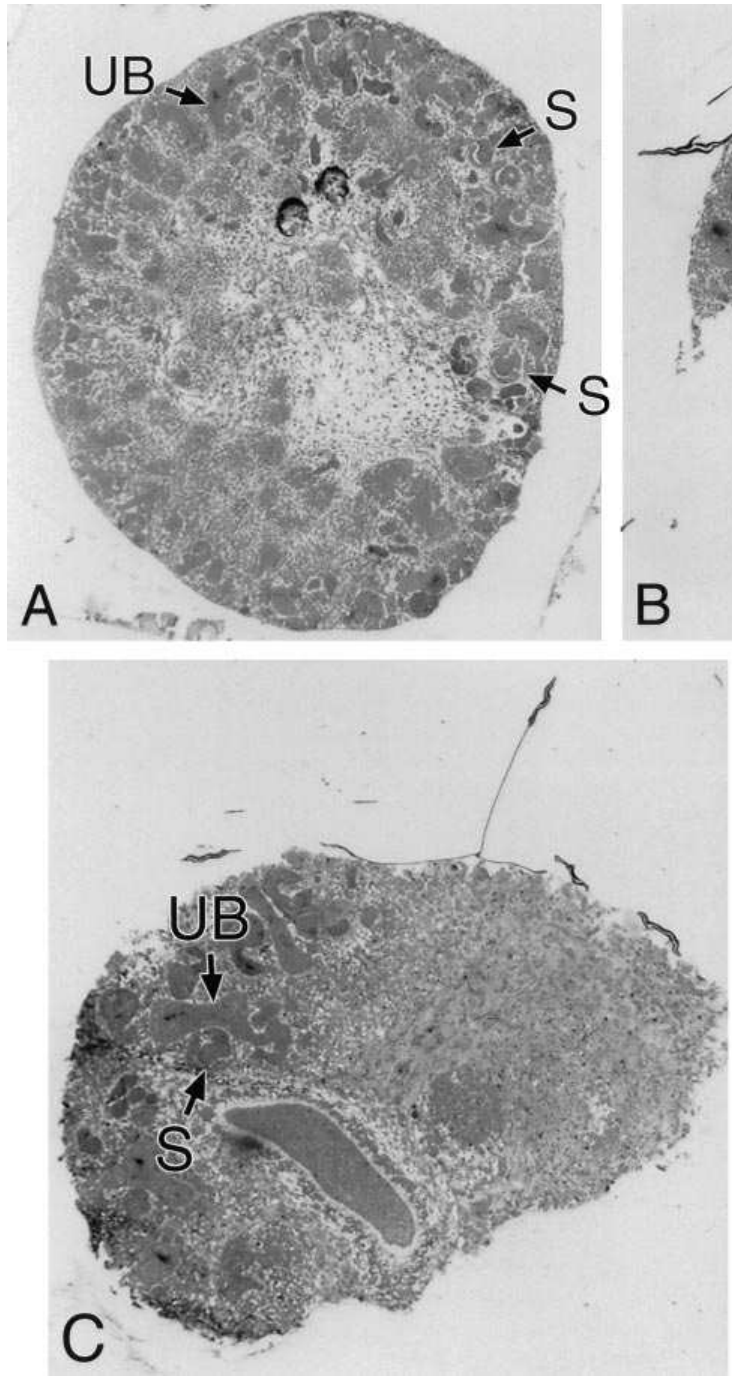

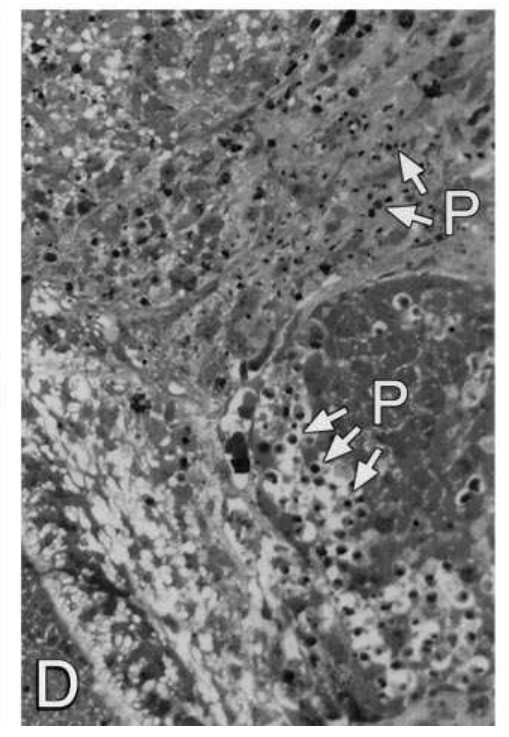

Figure 4. Photomicrographs of 12.5-d.p.c. metanephroi treated with Cux-1 ODNs. (A) $1-\mu \mathrm{m}$-thick section of a 12.5-d.p.c. metanephros cultured for $68 \mathrm{~h}$ in the presence of $5 \mu \mathrm{M}$ sense $C u x-1$ ODN (STr) and cationic liposomes showing normal nephron differentiation. (B) 1- $\mu \mathrm{m}$ thick section of a 12.5-d.p.c. metanephros dissected from the same embryonic litter cultured for $68 \mathrm{~h}$ in the presence of $5 \mu \mathrm{M}$ antisense Cux-1 ODN (ASTr) and cationic liposomes. The unlabeled arrow indicates a region of undifferentiated mesenchymal cells exhibiting increased numbers of pyknotic nuclei and apoptotic bodies. (C) 1- $\mu \mathrm{m}$ thick section of a 12.5-d.p.c. metanephros from the same litter as in $A$ and $B$, cultured for $68 \mathrm{~h}$ in the presence of $5 \mu \mathrm{M}$ antisense Cux-1 ODN (ASTr) and cationic liposomes. Note the extensive area devoid of ureteric buds or nephron structures that contains instead numerous cells with pyknotic nuclei and apoptotic bodies. $(D)$ Higher magnification image of the area shown in $(C)$. S, $S$-shaped body; UB, branch of the ureteric bud; P, pyknotic nuclei. $A$ and $B, 54 \times ; C, 69 \times ; D, 215 \times$. 

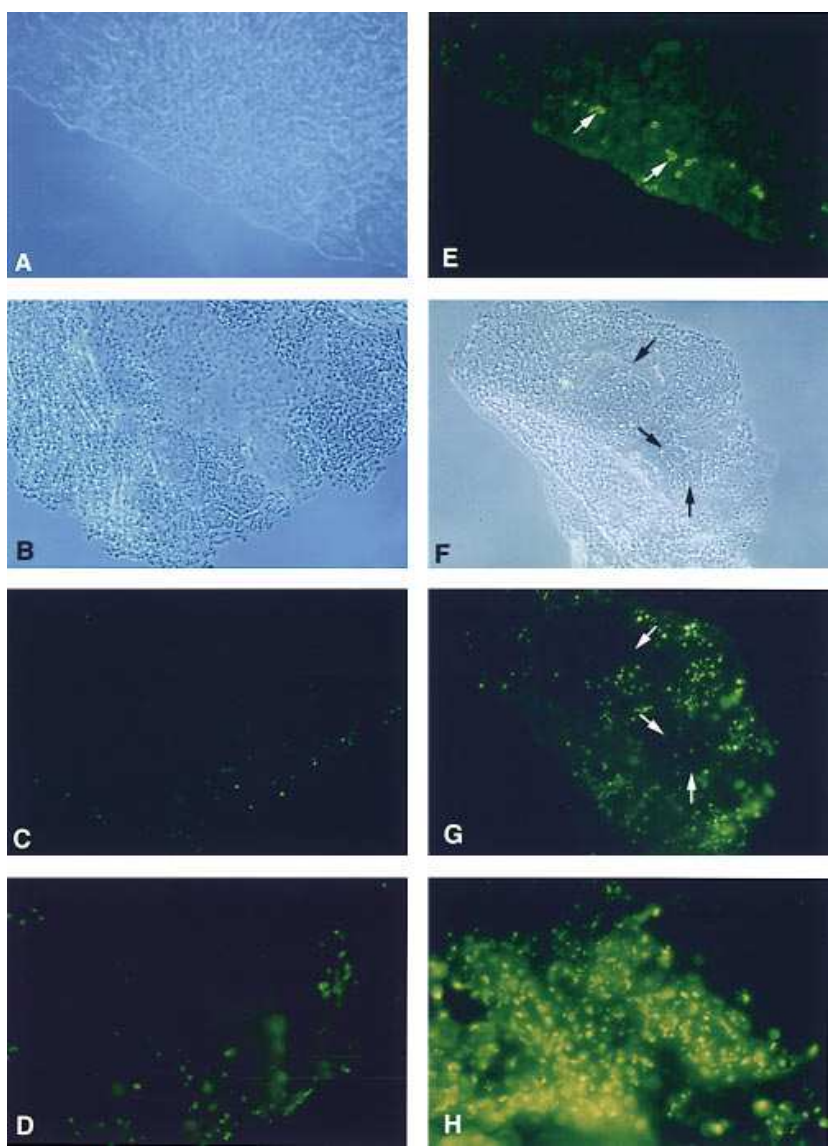

Figure 5. TUNEL analysis. Sections of kidney and ODN-treated metanephroi were labeled with fluorescein-12-dUTP using the TUNEL method. Phase contrast $(A)$ and fluorescence $(E)$ photomicrographs of a 3-d post partum kidney showing isolated labeled cells in the nephrogenic zone (arrows). Phase contrast $(B)$ and fluorescence $(C)$ photomicrographs of a metanephros after incubation for $68 \mathrm{~h}$ with a sense Cux-1 ODN matching the homeobox (SH). Phase contrast $(F)$ and fluorescence $(G)$ photomicrographs of a paired metanephros after incubation for $68 \mathrm{~h}$ with an antisense $C u x-1$ ODN (ASH). Note the marked increase in labeling of cells in the antisense ODN-treated metanephros, indicating increased apoptosis. Labeled cells are located in the mesenchyme and early nephron structures but are absent from the tubular epithelium ( $F$ and $G$, arrows). (D) Fluorescence photomicrograph of a metanephros after incubation for $68 \mathrm{~h}$ with a sense $C u x-1$ ODN matching the translation start codon (STr). $(H)$ Fluorescence photomicrograph of a paired metanephros after incubation with an antisense Cux-1 ODN (ASTr). Note the marked increase in labeled cells in the antisense ODN-treated metanephros. $A$, $B, C, E, F$, and $G, 56 \times ; D$ and $H, 111 \times$.

treated metanephroi, it was not possible to enumerate the number of labeled cells for comparison with sense ODNtreated metanephroi. However, labeling of antisense ODNtreated metanephroi was qualitatively much greater than sense ODN-treated metanephroi. Moreover, large areas of labeled cells, corresponding to the regions of cells containing pyknotic nuclei observed by light microscopy, were seen in metanephroi incubated with antisense ODNs, but were never observed in metanephroi incubated with sense ODNs. In addition, incubation with either antisense ODN (ASTr or AH) produced similar results. To determine the location of apoptotic cells, metanephroi were examined by fluorescence and phase contrast

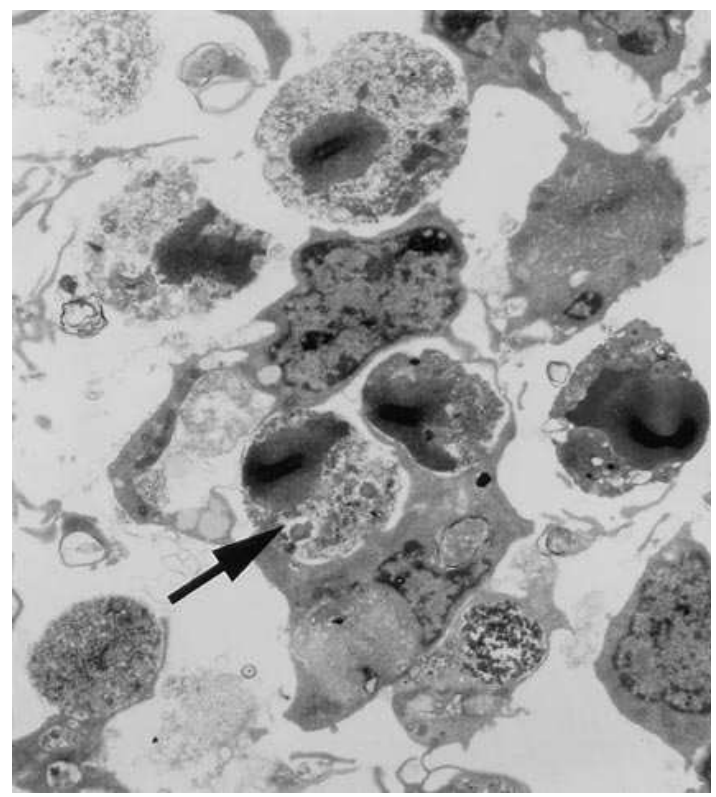

Figure 6. Identification of apoptotic changes by electron microscopy. Transmission electron micrograph of the antisense ODN-treated metanephros shown in Fig. 4 B. Note the presence of shrunken cells with distinct cell boundaries and uniformly condensed nuclei. The arrow indicates an apoptotic cell undergoing phagocytosis by a neighboring cell. $4,000 \times$.

microscopy (Fig. 5). Most of the apoptotic cells were present in the mesenchyme, although some labeled cells in $S$-shaped bodies and immature glomeruli were seen. No labeling was seen in tubular epithelial cells (Fig. 5 G, arrows).

Cell death due to apoptosis can be distinguished from necrosis by characteristic ultrastructural features (21). Initially, cells undergoing apoptosis exhibit cell shrinkage with crowding of organelles and condensation of chromatin, while the nuclear and plasma membranes remain intact. There is progressive condensation of chromatin with intense basophilic staining of the nuclei and nuclear fragmentation. Apoptotic bodies, which are small, membrane-bound bodies containing cytoplasmic and/or nuclear material are extruded into the intercellular space or undergo phagocytosis by neighboring cells. Accordingly, the regions of cell death observed in metanephroi treated with antisense ODNs were examined in greater detail by transmission electron microscopy. Fig. 6 shows that these regions contained shrunken cells in which the cell and nuclear boundaries were maintained and the nuclear chromatin was condensed into uniformly dense masses. In addition, numerous ovoid apoptotic bodies undergoing phagocytosis by neighboring cells were found in the mesenchyme (Fig. 6, arrow). Marginal clumping of loosely textured chromatin, rupture of the nuclear membrane, swelling of intracellular organelles, and cell swelling and lysis, which are characteristic of necrotic cell death, were not observed.

\section{Discussion}

Previous studies demonstrated that the murine $c u t$ homologue, Cux-1, was highly and transiently expressed during early metanephrogenesis (8). Since stage-dependent expression is charac- 
teristic of transcription factors that are important in nephrogenesis (e.g., WT-1, Pax-2), these studies suggested that $C u x-1$ may also be important during early nephrogenesis. To further evaluate whether $C u x-1$ was required for normal nephrogenesis, expression of Cux-1 was inhibited in vitro using antisense ODNs. The present study demonstrates that $\mathrm{Cux}-1$ antisense ODNs perturb normal nephrogenesis in embryonic kidney cultures. Compared with metanephroi incubated with Cux-1 sense ODNs, metanephroi incubated with $C u x$ - 1 antisense ODNs were $23 \%$ smaller and contained a marked increase in the number of cells undergoing apoptosis as evidenced by light microscopy, electron microscopy, and TUNEL analysis. The increased cell death was due to inhibition of $C u x-1$ and was not due to nonspecific cytotoxicity, as indicated by the following: (a) two different antisense ODNs targeting distinct regions of the transcript produced an identical phenotype, $(b)$ cell death was not increased in metanephroi incubated with two different sense ODNs, (c) a decrease in Cux-1 mRNA levels was observed in metanephroi treated with antisense ODNs, but not sense ODNs, and $(d)$ cell death had characteristic biochemical and morphologic features of apoptosis rather than nonspecific tissue necrosis.

Metanephric explants incubated with antisense $C u x$ - 1 ODNs continue to exhibit branching of ureteric buds and development of epithelial structures including $S$-shaped bodies. Thus, Cux -1 antisense ODNs do not completely block nephrogenic induction. However, Cux-1 antisense ODN-treated metanephroi also contain regions of mesenchymal cell death that are devoid of epithelial structures. In contrast, antisense inhibition of $P a x-2$ results in a complete block of nephrogenic induction, absence of induced nephric structures, and decreased ureteric branching, but no evidence of increased apoptosis (12). Thus, the effects of antisense inhibition of Cux-1 and $P a x-2$ are distinct, suggesting that these transcription factors have different functions during nephrogenesis.

Apoptosis occurs during normal kidney development. Coles et al. (20) have reported that as many as 3\% of cells in the nephrogenic zone and a smaller number of cells in the medullary papilla may be undergoing apoptosis at any given time during nephrogenesis. It is likely that apoptosis plays an important role in the selection of cells available for induction during mammalian nephrogenesis. Although apoptosis is present during normal nephrogenesis, Cux-1 antisense ODNtreated metanephroi show an abnormal increase in the number of apoptotic cells compared with sense ODN-treated metanephroi. Apoptotic cells were found predominantly in metanephric mesenchymal cells and to a lesser degree in early stage nephrons (renal vesicles and $S$-shaped bodies). No apoptotic cells were observed in tubular epithelial cells. Compared with epithelial cells, metanephric mesenchymal cells may be especially sensitive to inhibition of $C u x-1$ since $C u x-1$ is most highly expressed in this cell type and these cells are more permeant to antisense ODNs $(8,22)$. Increased apoptosis has been observed after other experimental manipulations of kidney development. For example, $10-50 \%$ of the metanephric blastemal cells in WT-1 null mice are undergoing apoptosis, compared with $1 \%$ in wild-type embryos (4). Incubation of metanephric cultures with neutralizing antibodies to hepatocyte growth factor causes an increase in cell death in the renal mesenchyme (23). Metanephric mesenchymal cells placed in transfilter culture undergo apoptosis in the absence of inducing tissue, and cell death can be inhibited by epidermal growth factor
(24). These observations suggest that increased cell death in the developing kidney can result from both cell- and non-cellautonomous defects.

The expression of $C u x-1$ in the developing kidney is inversely related to the degree of nephron differentiation (8). Transfection studies have shown that other mammalian cut homologues function as transcriptional repressors of genes specifying terminal differentiation in multiple cell lineages $(7,25$ 27). These observations suggest that Cux-1 may function as a transcriptional repressor to inhibit terminally differentiated gene expression during early stages of nephrogenesis. Because the genes that are regulated by Cux-1 have not been identified in the developing kidney, the mechanism by which inhibition of Cux-1 leads to increased apoptosis remains unknown. However, the observation that inhibition of Cux-1 results in increased apoptosis is consistent with the notion that regulation of Cux-1 is required for normal nephron differentiation. One possibility is that inhibition of Cux-1 disturbs the normal relationship between cell proliferation and cell differentiation, which may result in apoptosis as a default pathway (28).

In summary, incubation of murine metanephroi with two different $C u x-1$ antisense ODNs produces a marked increase in the number of cells undergoing apoptosis, predominantly in metanephric mesenchyme. These results suggest that proper regulation of $C u x-1$ expression may be necessary for normal kidney development. In addition, we report the first use of cationic liposomes to deliver ODNs to metanephroi in vitro. The use of cationic liposomes may reduce the quantity of phosphorothioate-coupled antisense ODN that is required to inhibit gene expression and thereby decrease the potential toxic effects of phosphorothioate ODNs.

\section{Acknowledgments}

We thank Sue Ann Mentone for excellent technical assistance, Dr. Robert Reilly for providing protocols for RT-PCR, and Dr. Greg Vanden Heuvel for critically reviewing the manuscript.

This study was supported by National Institutes of Health grant RO1 DK-45678. S.E. Quaggin is the recipient of a Clinician-Scientist Award from the Medical Research Council of Canada. P. Igarashi is an Established Investigator of the American Heart Association.

\section{References}

1. Saxén, L. 1987. Organogenesis of the Kidney. Cambridge University Press, Cambridge, UK. 173 pp.

2. Bard, J.B.L., J.E. McConnell, and J.A. Davies. 1994. Towards a genetic basis for kidney development. Mech. Dev. 48:3-11.

3. Igarashi, P. 1994. Transcription factors and apoptosis in kidney development. Curr. Opin. Nephrol. Hypertens. 3:308-317.

4. Kreidberg, J.A., H. Sariola, J.M. Loring, M. Maeda, J. Pelletier, D. Housman, and R. Jaenisch. 1993. WT-1 is required for early kidney development. Cell. 74:679-691.

5. Torres, M., E. Gomez-Pardo, G.R. Dressler, and P. Gruss. 1995. Pax-2 controls multiple steps of urogenital development. Development (Camb.). 121: 4057-4065.

6. Hatini, V., S.O. Huh, D. Herzlinger, V.C. Soares, and E. Lai. 1996. Essential role of stromal mesenchyme in kidney morphogenesis revealed by targeted disruption of Winged Helix transcription factor BF-2. Genes Dev. 10: 1467-1478.

7. Valarché, I., J.P. Tissier-Seta, M.R. Hirsch, S. Martinez, C. Goridis, and J.F. Brunet. 1993. The mouse homeodomain protein Phox2 regulates Ncam promoter activity in concert with Cux/CDP and is a putative determinant of neurotransmitter phenotype. Development (Camb.). 119:881-896.

8. Vanden Heuvel, G.B., R. Bodmer, K.R. McConnell, G.T. Nagami, and P. Igarashi. 1996. Expression of a cut-related homeobox gene in developing and polycystic mouse kidney. Kidney Int. 50:453-461.

9. Saxén, L., O. Koskimies, A. Lahti, H. Miettinen, J. Rapola, and J. Wartiovaara. 1968. Differentiation of kidney mesenchyme in an experimental 
model system. Adv. Morphog. 7:251-293.

10. Avner, E.D., D. Ellis, T. Temple, and R. Jaffe. 1982. Metanephric development in serum-free organ culture. In Vitro (Rockville). 18:675-682.

11. Avner, E.D., N.P. Piesco, W.E. Sweeney, and D. Ellis. 1988. Renal epithelial development in organotypic culture. Pediatr. Nephrol. 2:92-99.

12. Rothenpieler, U.W., and G.R. Dressler. 1993. Pax-2 is required for mesenchyme-to-epithelium conversion during kidney development. Development (Camb.). 119:711-720.

13. Sariola, H., M. Saarma, K. Sainio, U. Arumae, J. Palgi, A. Vaahtokari, I. Thesleff, and A. Karavanov. 1991. Dependence of kidney morphogenesis on the expression of nerve growth factor receptor. Science (Wash. DC). 254:571573.

14. Yeger, H., D. Forget, J. Alami, and B.R.G. Williams. 1996. Analysis of WT1 gene expression during mouse nephrogenesis in organ culture. In Vitro Cell. Dev. Biol. 32:496-504.

15. Theiler, K. 1972. The House Mouse: Development and Normal Stages From Fertilization to 4 Weeks of Age. Springer-Verlag, New York. 168 pp.

16. Chomczynski, P., and N. Sacchi. 1987. Single-step method of RNA isolation by acid guanidinium thiocyanate-phenol-chloroform extraction. Anal. Biochem. 162:156-159.

17. Gavrieli, Y, Y. Sherman, and S.A. Ben-Sasson. 1992. Identification of programmed cell death in situ via specific labeling of nuclear DNA fragmentation. J. Cell Biol. 119:493-501.

18. Sgonc, R., G. Boeck, H. Dietrich, J. Gruber, H. Recheis, and G. Wick. 1994. Simultaneous determination of cell surface antigens and apoptosis. Trends Genet. 10:41-42.

19. Wyllie, A.H., R.G. Morris, A.L. Smith, and D. Dunlop. 1984. Chromatin cleavage in apoptosis: association with condensed chromatin morphology and dependence on macromolecular synthesis. J. Pathol. 142:67-77.

20. Coles, H.S.R., J.F. Burne, and M.C. Raff. 1993. Large-scale normal cell death in the developing rat kidney and its reduction by epidermal growth factor. Development (Camb.). 118:777-784.

21. Wyllie, A.H., J.F.R. Kerr, and A.R. Currie. 1980. Cell death: the significance of apoptosis. Int. Rev. Cytol. 68:251-306.

22. Rothenpieler, U.W., and G.R. Dressler. 1993. Differential distribution of oligodeoxynucleotides in developing organs with epithelial-mesenchymal interactions. Nucleic Acids Res. 21:4961-4966.

23. Woolf, A.S., M. Kolatsi-Joannou, P. Hardman, E. Andermarcher, C. Moorby, L.G. Fine, P.S. Jat, M.D. Noble, and E. Gherardi. 1995. Roles of hepatocyte growth factor/scatter factor and the met receptor in the early development of the metanephros. J. Cell Biol. 128:171-184.

24. Koseki, C., D. Herzlinger, and Q. Al-Awqati. 1992. Apoptosis in metanephric development. J. Cell Biol. 119:1327-1333.

25. Lievens, P.M.J., J.J. Donady, C. Tufarelli, and E.J. Neufeld. 1995. Repressor activity of CCAAT displacement protein in HL-60 myeloid leukemia cells. J. Biol. Chem. 270:12745-12750.

26. Andres, V., B. Nadal-Ginard, and V. Mahdavi. 1992. Clox, a mammalian homeobox gene related to Drosophila cut, encodes DNA-binding regulatory proteins differentially expressed during development. Development (Camb.). 116:321-334.

27. Dufort, D., and A. Nepveu. 1994. The human Cut homeodomain protein represses transcription from the $c$-myc promoter. Mol. Cell. Biol. 14:42514257.

28. Thompson, C.B. 1995. Apoptosis in the pathogenesis and treatment of disease. Science (Wash. DC). 267:1456-1462. 